\title{
The diagnosis of ADHD in Australian children: current paediatric practice and parent perspective
}

Original Article

Authors: Daryl Efron, ${ }^{1,2,3}$ Emma Sciberras, ${ }^{1,2,3}$ Harriet Hiscock,,${ }^{1,2,3}$ Brad Jongeling,,${ }^{4,5}$ Kate Lycett ${ }^{1,2,3}$ Matthew Bisset, ${ }^{1}$ Grant Smith $^{6}$

\section{Institutions:}

${ }^{1}$ Murdoch Childrens Research Institute, Parkville, VIC 3052

${ }^{2}$ Centre for Community Child Health, The Royal Children's Hospital, Parkville, VIC 3052

${ }^{3}$ The University of Melbourne, Parkville, VIC 3010

${ }^{4}$ Child Development Service, Joondalup, WA 6919

${ }^{5}$ The University of Western Australia, Perth, WA 6009

${ }^{6}$ Telethon Kids Institute, The University of Western Australia, Crawley, WA 6009

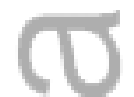

Running heading: Diagnosing ADHD in Australian children

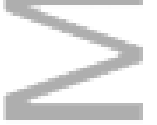

Key words:

attention-deficit hyperactivity disorder, quality of health care,

paediatrics, guideline adherence, behavioural medicine

\section{Correspondence to:}

Daryl Efron

Centre for Community Child Health, The Royal Children's Hospital, Flemington Road, Parkville Vic 3052, Australia.

$$
\text { P + } 61393454563 \text { F +61393454751 email: daryl.efron@ @rch.org.au }
$$

This is the author manuscript accepted for publication and has undergone full peer review but has not been through the copyediting, typesetting, pagination and proofreading process, which may lead to differences between this version and the Version of Record. Please cite this article as doi: 
Conflicts of interest: None

Word count: 2,500 (Abstract: 248)

\section{Abbreviations}

ADHD - Attention-Deficit/Hyperactivity Disorder

DSM - Diagnostic and Statistical Manual of Mental Disorders

ICD - International Classification of Diseases

APA - American Academy of Pediatrics

US - United States

VIC - Victoria

WA - Western Australia

SEIFA - Socio-Economic Indexes for Areas

SDQ - Strengths and Difficulties Questionnaire

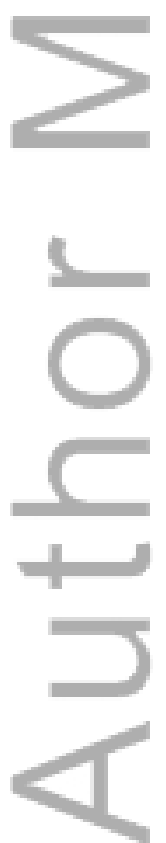

This article is protected by copyright. All rights reserved. 


\begin{abstract}
$\operatorname{Aims}$

In a sample of newly diagnosed children with ADHD, to examine 1) paediatrician assessment and management practices, 2) previous assessments and interventions, 3) correspondence between parent-report and paediatrician identification of comorbidities; and 4) parent agreement with diagnosis of ADHD.

\section{Methods}

Design: Cross-sectional, multi-site practice audit with questionnaires completed by paediatricians and parents at the point of ADHD diagnosis. Setting: Private/public paediatric practices in Western Australia and Victoria, Australia. Main outcome measures: Paediatricians: Elements of assessment and management were indicated on a study-designed data form. Parents: ADHD symptoms and comorbidities were measured using the Conners 3 ADHD Index and Strengths and Difficulties Questionnaire, respectively. Sleep problems, previous assessments and interventions, and agreement with ADHD diagnosis were measured by questionnaire.

\title{
Results
}

24 paediatricians participated, providing data on 137 patients $(77 \%$ male, mean age 8.1 years). Parent and teacher questionnaires were used in $88 \%$ and $85 \%$ of assessments respectively. Medication was prescribed in $75 \%$ of cases. Comorbidities were commonly diagnosed $(70 \%)$; however, the proportion of patients identified by paediatricians with internalising problems (18\%), externalising problems (15\%) and sleep problems (4\%) was less than by parent report $(51 \%, 66 \%, 39 \%)$. One in seven parents did not agree with the diagnosis of ADHD.

\section{Conclusions}

Australian paediatric practice in relation to ADHD assessment is generally consistent with best practice guidelines; however, improvements are needed in relation to the routine use of questionnaires and the identification of comorbidities. A proportion of parents do not agree with the diagnosis of ADHD made by their paediatrician. 


\section{Key terms}

ADHD, quality of health care, paediatrics, guideline adherence, behavioural medicine

\section{What is already known}

- $\mathrm{ADHD}$ is the most common diagnosis in children attending general and community paediatricians in Australia

- "International guidelines articulate consistent recommendations for best practice in the diagnosis and management of ADHD, but Australian adherence with best practice is unknown.

- Parent agreement with paediatrician diagnosis of ADHD and comorbidities is similarly unknown.

\section{What this study adds}

- Australian paediatricians' diagnostic and management practices are broadly consistent with international guidelines.

- However, paediatricians under-identified externalising and externalising comorbidities, as well as sleep problems, compared with parental report. One in seven parents did not agree with the diagnosis.

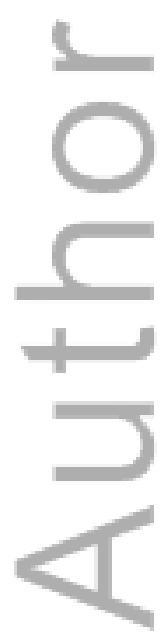

This article is protected by copyright. All rights reserved. 


\section{INTRODUCTION}

Attention deficit hyperactivity disorder (ADHD) is a prevalent developmental disorder usually managed with long-term medication treatment. The quality of clinical diagnosis and treatment is therefore highly important in providing safe and optimal care for these patients. Recent editions of the International Classification of Diseases (ICD) ${ }^{1}$ and the Diagnostic and Statistical Manual of Mental Disorders (DSM) ${ }^{2}$ have seen a convergence in the definition and assessment requirements of Hyperkinetic Disorder (ICD) and ADHD (DSM), respectively. Both demand evidence of cross-situational impairment (home and school) and DSM-5 specifically recommends gathering independent information "confirming substantial symptoms across settings" from informants "who have seen the individual in those settings" (i.e. parents and teachers). ${ }^{2}$ Further, DSM-5 explicitly recognizes that comorbidities are the rule rather than the exception in ADHD and must be identified and addressed. $^{2}$

A number of clinical practice guidelines have been published internationally with the aim of improving the reliability of diagnosis, comprehensiveness of clinical assessment and standardization of management of children and adolescents referred for evaluation for ADHD. ${ }^{3-6}$ The Australian National Health and Medical Research Council recently published Clinical Practice Points articulating recommended best practice in the diagnosis and management of ADHD. ${ }^{7}$ These guidelines vary in structure and emphasis, yet share the following key recommendations: a) use of standardized behaviour rating scales; b) obtaining information from multiple sources, specifically including teachers; c) evaluation for comorbid developmental, physical and mental health diagnoses - including referral for additional assessment, as indicated; and d) monitoring of weight, height and blood pressure in children taking stimulant medication.

As paediatricians are the main physicians diagnosing ADHD in Australia, ${ }^{8}$ it is important to examine their diagnostic and management practice, particularly given the everpresent controversy surrounding ADHD and the use of stimulant medications. Existing research into paediatricians' ADHD diagnostic practice has occurred mostly in North America, with a large study of primary care paediatric consultations $(n=401)$ conducted in 1999 across the United States (US), Canada and Puerto Rico finding that behavioural questionnaires were only used in $37 \%$ of assessments for attentional and hyperactivity problems, indicating a lack of standardization of diagnostic practice. ${ }^{9}$ A 2004 survey of 
primary care physicians in Michigan found high awareness of American Academy of Pediatrics (AAP) clinical guidelines on ADHD (91\% of paediatricians, 60\% of family physicians), but variation in practice quality in relation to diagnostic procedures, investigations, use of behavioural therapy and follow-up intervals. ${ }^{10}$

A study from New York found that the majority of paediatricians did not follow AAP guidelines for preschool-aged children diagnosed with ADHD. ${ }^{11}$ A recent large retrospective chart review of ADHD patients in 50 US paediatric practices (188 paediatricians) found that parent-and teacher-rating scales were used in assessment in just over half of 1594 cases and only $13 \%$ received psychosocial treatment. ${ }^{12}$ In the United Kingdom, an audit of the assessment and initial management of 63 children seen in a multi-disciplinary ADHD clinic found that performance was generally consistent with the relevant National Institute for Health and Care Excellence guidelines; in particular, shared-care with general practitioners was generally established successfully following initiation of medication. ${ }^{13}$ There has been little published research on ADHD assessment processes in Europe or Australasia. We recently published the first large-scale description of the clinical profile of children with ADHD attending paediatricians in Australia $(n=1528$, mean age 9.1 years $),{ }^{14}$ finding that comorbidities appeared to be under-identified and raising questions about the rigor of evaluation processes. However, these data were drawn from a large audit of children attending paediatricians for any reason, including both new and continuing patients, with data solely from paediatricians.

Most previous work examining adherence to ADHD guidelines has neglected parent views. Parents of children with ADHD symptoms often experience confusion, worry and doubt in relation to the diagnosis and associated medication treatment, ${ }^{15}$ and sometimes perceive health professionals to be dismissive of their concerns. ${ }^{16}$ They do not always agree with diagnoses made or treatment recommended (particularly fathers), ${ }^{16}, 17$ and commonly use complementary therapies. ${ }^{18} \mathrm{~A}$ better understanding of parental attitudes regarding assessment, diagnosis and treatment of ADHD may lead to more sensitive communication of assessment findings and therapeutic recommendations, which in turn may result in improved adherence to treatment and better outcomes.

This study investigated how current paediatric practice aligns with key recommendations from international guidelines for the assessment and treatment of ADHD, and also integrated parents' perspective. The aims were to examine, in a sample of children newly diagnosed with ADHD: 
1. paediatrician assessment and initial management practices;

2. previous assessments and interventions;

3. correspondence between parent-report and paediatrician identification of comorbidities; and

4. parent agreement with the diagnosis of ADHD.

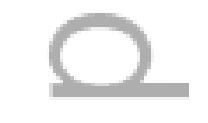

-

\section{MATERIALS AND METHODS}

Setting: 30 public and private paediatric practices in Victoria (VIC; $n=9$ ) and Western Australia (WA; n=21).

Design: Multi-informant questionnaires, collected at or around the point of diagnosis (Vic: Sep 2012-Jan 2013; WA: May 2013-Feb 2014).

Participants: Paediatricians and parents of children and adolescents, aged 3 to 17 years, with newly diagnosed ADHD.

Procedure: Paediatricians were identified through convenience sampling, and asked to complete a short questionnaire about their practice. Participating paediatricians were asked to complete a study-designed data form describing their assessment and initial management (Aim 1) for each patient newly diagnosed with ADHD seen during the study period. Paediatricians were requested to inform parents about the study and to seek consent for their contact details to be passed on to the study team. Parents who provided contact details were invited to complete a questionnaire. The parent questionnaire collected information about previous assessments and interventions (Aim 2), parent report of the child's internalising, externalising and sleep problems (Aim 3) and parent agreement with the diagnosis of ADHD (Aim 4).

Measures: All measures are detailed in Table 1. In addition, parents provided child and family demographic information, and socioeconomic status was estimated using the postcode level Socio-Economic Indexes for Areas (SEIFA) disadvantage Index (mean 1000, SD 100). ${ }^{19}$

Data analyses: All analyses were conducted using Stata version 13. Descriptive statistics were conducted. The characteristics of participants were compared to non- 
participants using chi-square and t-tests. For the paediatrician-reported data $95 \%$ confidence intervals were calculated adjusting for paediatrician clustering.

Ethics: Study approval was granted by the Human Research Ethics Committees of The Royal Children's Hospital, Melbourne, VIC (\#32126) and The Princess Margaret Hospital in Perth, WA (2041/EP).

=

\section{RESULTS}

Sample: Paediatricians and patients

Of the 54 paediatricians approached (VIC: 15; WA: 39), 41 agreed to participate (VIC: 12; WA: 29; 30 practices), of whom 24 (59\%; VIC: 7; WA: 17; 22 practices) provided data on at least one newly diagnosed patient with ADHD. Half of the referring paediatricians were male and $78 \%$ were aged between 35-54 years. The majority worked in a sole practitioner model, while $33 \%$ worked within a multidisciplinary team. Referring paediatricians were comparable to non-referring paediatricians in terms of the demographics described above (all $\mathrm{p}>0.05$ ).

Data were provided on 137 newly diagnosed patients (Table 2). Patients came from less disadvantaged families compared to population norms (sample SEIFA mean 1036.9, SD 48.8; $\mathrm{p}<0.001$ compared to SEIFA norms). The mean age of patients was 8.1 years (SD 2.7, range $3-17$ ) and $77 \%$ were male. ADHD Combined subtype was most common (50.4\%), followed by Predominantly Inattentive subtype (29.9\%) and Predominantly Hyperactive/Impulsive subtype (19.7\%). Three quarters were from WA and most were recruited from private practices $(77.4 \%)$, followed by child development centres $(18.3 \%)$ and public hospital outpatient clinics (2.9\%). Recruited patients were not evenly distributed across paediatricians, with $60 \%$ referred by five paediatricians.

Paediatrician assessment and initial management (see Table 2)

Paediatricians reported using parent $(\mathrm{P})$ and teacher $(\mathrm{T})$ questionnaires in $88 \%$ and $85 \%$ of patients, respectively. However this differed between the two states (WA: P 95\%, T 92\%; Vic: P 63\%, T 64\%). Paediatricians identified $26.3 \%$ of patients as having a comorbid learning disability (95\% CI 18.1, 36.5), 18.3\% (95\% CI: 8.3, 35.6) an internalising disorder, 
14.6\% (95\% CI: 8.2, 24.6) an externalising disorder and 3.7\% (95\% CI: 1.3, 10.2) a sleep disorder.

Medication was prescribed for $75 \%$ of patients, with methylphenidate being the most commonly prescribed medication. Height and weight were recorded in $91 \%$ and $95 \%$ of consultations, respectively. Of those patients prescribed medication, only three did not have their weight measured and four did not have height measured.

Parent report of previous assessments, child symptoms, and agreement with diagnosis (see Table 3)

Of the 137 patients, $80(58 \%)$ parents completed questionnaires, of which $54(68 \%)$ were cases referred by the five principal referring paediatricians. There were no differences between responders and non-responders in terms of child age, gender, family neighbourhood disadvantage, paediatrician-diagnosed externalising comorbidities or sleep disorders. However, responders were more likely to have a paediatrician-diagnosed internalising comorbidity compared to non-responders ( $24 \%$ vs. $11 \%$; $\mathrm{p}=0.05)$. On average, questionnaires were completed 43 days after the consultation.

Parents had previously consulted a professional about the child's behaviour in $91 \%$ of cases (most commonly paediatrician, psychologist, and/or general practitioner) and twothirds of parents had researched the diagnostic criteria for ADHD prior to diagnosis. Use of natural and complementary therapies was reported by $46 \%$ and dietary restrictions by $49 \%$ (66\% used either). The mean interval from parents' first concerns to paediatric assessment was about two years.

Parents reported that the diagnosis was made in one, two, or three or more visits in one-third of cases each. The majority of parents (85\%) "Completely or somewhat agreed" with their child's diagnosis of ADHD. A higher proportion of parents reported internalising problems (51\%), externalising problems (66\%) and sleep problems (39\%) compared to paediatricians $(18 \%, 15 \%, 4 \%)$.

\section{DISCUSSION}

In this novel, multi-informant, point-of-diagnosis study of children with ADHD, paediatricians' assessment and initial management practices were generally consistent with recommendations from clinical guidelines. However, in a substantial minority of cases there 
were deviations from recommended best practice. In particular, the diagnosis was made without the use of standardized parent or teacher questionnaires in $12 \%$ and $15 \%$ of cases, respectively. Adherence to this aspect of the guidelines was better in WA than Victoria. This may be due to many participating WA paediatricians working in child development centres which have standardized assessment procedures; these paediatricians may carry such procedures over into their private practice. Given the high prevalence of this condition these findings raise concern that some children may be incorrectly diagnosed, leading to unnecessary medication treatment.

The diagnosis was made in a single consultation in one third of cases, with an average consultation duration of 48 minutes. This appears brief to gather all relevant data necessary to make the diagnosis. However, it is possible the paediatricians had known the child previously in some cases and so the assessment process was able to be expedited. Although there is no gold standard recommendation for either number or duration of consultations to diagnose ADHD, parents are sometimes dissatisfied with perceived overly brief assessment and diagnosis. $^{15}$

Clinical range scores on the emotional and conduct problems scales of the SDQ have been shown to correlate highly with gold standard clinical assessment of internalising and externalising disorders. ${ }^{20}$ In this study, paediatricians' identification of both internalising and externalising comorbidities was low in comparison with the proportion of children with clinical scores on these scales by parent report (SDQ). Given that the children of nonresponding parents were less likely than those of responders to have a paediatrician-reported internalising disorder, this difference may actually be an underestimate. Furthermore, whereas over one third of parents reported that their child had a significant sleep problem, paediatricians identified a comorbid sleep disorder in only $4 \%$ of cases. These findings are consistent with our audit of 199 paediatricians' practice, ${ }^{14}$ providing further evidence of the need for paediatricians to systematically evaluate for comorbidities, which are present in the majority of children with ADHD and require concurrent management.

Prior to paediatric assessment over $90 \%$ of parents had accessed some form of professional help for their child's behaviour or learning, substantially higher than that previously reported by parents of children with ADHD identified in an Australian national survey. ${ }^{21}$ Furthermore, two thirds had researched the diagnostic criteria for ADHD. These findings suggest Australian parents (at least those who access a paediatrician) have become more proactive in seeking assistance for their child without waiting for a formal diagnosis, 
and in finding information to inform the diagnosis. There was however an average 15 month interval from initial parental concern to paediatric referral, which may reflect some reluctance to seek medical assessment and treatment.

In this study parents did not agree with the diagnosis in $15 \%$ of cases. It is known that agreement between parents and teachers reporting of children's ADHD symptoms is generally modest. ${ }^{22}$ However, there has been little exploration of parental agreement with ADHD diagnosis, so this represents a novel finding. Given that acceptance of the diagnosis is a key factor in adherence with recommended psychotropic medication treatment in children and adolescents, ${ }^{23}$ our study highlights the importance of exploring parental acceptance of the diagnosis of ADHD in order to work together to achieve good outcomes. The high proportion of parents who reported using complementary and dietary interventions, while consistent with previous studies, ${ }^{24}$ underlines the importance of paediatricians enquiring about these interventions and informing parents about evidence for their effectiveness and potential sideeffects, as well as the opportunity cost for applying conventional interventions.

The rate of adherence to best practice guidelines in this study was higher than that reported in the US. ${ }^{12}$ This discrepancy may be explained by the fact that, in contrast to American paediatricians (primary care), Australian paediatricians function as secondary care providers i.e. specialists who can only see patients on referral from a general practitioner. Encouragingly, adherence with guidelines in this study was higher than reported in two previous postal surveys of Australian parents of children with $\mathrm{ADHD}^{25,8}$ suggesting that ADHD practice has improved in Australia, although further improvements are needed. A quality improvement intervention in ADHD assessment and management for primary care providers has been associated with improved adherence to evidence-based guidelines ${ }^{26}$ as well as reduced ADHD symptoms. ${ }^{27}$

This study had the important design strengths of data collection being at or around the point of diagnosis and from two sources, paediatricians and parents. The major limitation was potential sampling bias, as the patients were recruited by a sample of self-selected paediatricians in Victoria and Western Australia, and so the findings may not be generalisable Australia-wide. Furthermore, a large proportion of the patients were referred by a small number of paediatricians. However, we attempted to address this by calculating 95\% confidence intervals adjusted for clustering when presenting our paediatrician-reported data, which showed that some of our findings had relatively low precision and so require replication, in particular the prevalence of identified comorbidities. Paediatricians knew that 
they were being assessed and may have altered their practice accordingly or selectively referred patients where their diagnostic process followed best-practice guidelines. Furthermore, participating paediatricians may have been more likely to be familiar with ADHD clinical guidelines, thus our findings may under-estimate non-adherence with recommended best practice.

In summary, this study identified areas for improvement in relation to the diagnosis and initial management of ADHD; in particular, the routine use of parent and teacher questionnaires, which showed regional variation, and the identification of comorbidities. Furthermore, we found that parents of children with ADHD demonstrate initiative in seeking professional help and having their children assessed; however, a proportion do not agree with the diagnosis, reinforcing the need for paediatricians to continue working to engage parents of children with ADHD as partners in the management of their children.

\section{ACKNOWLEDGEMENTS}

Author A is funded by a Career Development Award from his institute. Funding support was provided by the Department of Health, Western Australia under the WA Health Projects for Telethon Institute of Child Health Research Service Agreement. The researchers acknowledge the Australian National Health and Medical Research Council for salary support for Authors B and C. The funding agencies were not involved in the study conduct or interpreting results. We would like to thank all families and paediatricians who participated in this study. Some study data were collected and managed using REDCap (Research Electronic Data Capture) electronic data capture tools. REDCap is a secure, web-based application designed to support data capture for research studies.

This article is protected by copyright. All rights reserved. 


\section{REFERENCES}

1. World Health Organization. The ICD-10 classification of mental and behavioural disorders : diagnostic criteria for research. Geneva: World Health Organization; 1993.

2. American Psychiatric Association. Diagnostic and statistical manual of mental disorders (5th ed.). Arlington, VA: American Psychiatric Publishing; 2013.

3. NICE clinical guideline 72. Attention deficit hyperactivity disorder: Diagnosis and management of ADHD in children, young people and adults. NHS 2008.

4. American Academy of Child and Adolescent Psychiatry. Practice Parameter for Attention deficit hyperactivity disorder. J Am Acad Child Adolesc Psychiatry. 2007:46:894-921.

5. = Subcommitee on attention-deficit/hypercativity disorder steering commitee on quality improvment and mangement. ADHD: clinical practice guideline for the diagnosis, evaluation, and treatment of attention-deficit/hyperactivity disorder in children and adolescents. Pediatrics. 2011;128(5):1007-22.

6. Hiscock H, Roberts G, Efron D, Sewell JR, Bryson HE, Price AM, et al. Children Attending Paediatricians Study: a national prospective audit of outpatient practice from the Australian Paediatric Research Network. Med J Aust. 2011;194(8):392-7.

7. Council NHaMR. Clinical Practice Points on the Diagnosis, Assessment and Management of ADHD in Children and Adolescents. NHMRC, 2012.

8. Concannon PE, Tang YP. Management of attention deficit hyperactivity disorder: a parental perspective. J Paediatr Child Health. 2005;41(12):625-30.

9. Wasserman RC, Kelleher KJ, Bocian A, Baker A, Childs GE, Indacochea F, et al. Identification of attentional and hyperactivity problems in primary care: a report from pediatric research in office settings and the ambulatory sentinel practice network. Pediatrics. 1999;103(3):E38.

10. Rushton JL, Fant KE, Clark SJ. Use of practice guidelines in the primary care of children with attention-deficit/hyperactivity disorder. Pediatrics. 2004;114(1):e23-8.

11. Chung J SS, Meryash D, Gutman A, Adesman A. . Medication Management of Preschool ADHD by Pediatric Sub-Specialists: Non-Compliance with AAP Clinical Guidelines. Pediatric Academic Societies; Washington, D.C.2013.

12. Epstein JN, Kelleher KJ, Baum R, Brinkman WB, Peugh J, Gardner W, et al. Variability in ADHD Care in Community-Based Pediatrics. Pediatrics. 2014;134(6):1136-43.

13. Allen R GH. An audit of an ADHD Assessment Clinic in light of NICE guidelines. Clin Govern Int J. 2004;9(3):167 - 71.

14. Efron D, Davies S, Sciberras E. Current Australian pediatric practice in the assessment and treatment of ADHD. Acad Pediatr. 2013;13(4):328-33.

15. Charach A, Skyba A, Cook L, Antle BJ. Using stimulant medication for children with ADHD: What do parents say? A brief report. Journal of the Canadian Academy of Child and Adolescent Psychiatry. 2006;15(2):75.

16. DosReis S, Barksdale CL, Sherman A, Maloney K, Charach A. Stigmatizing experiences of parents of children with a new diagnosis of ADHD. Psychiatr Serv. 2010;61(8):811-6.

17. Singh I. Boys will be boys: Fathers' perspectives on ADHD symptoms, diagnosis, and drug treatment. Harvard Rev Psychiat. 2003;11(6):308-16.

18. Weber W, Newmark S. Complementary and alternative medical therapies for attention-deficit/hyperactivity disorder and autism. Pediatr Clin North Am. 2007 54(6):983-1006. 
19. Australian Bureau of Statistics. Census of Population and Housing: Socio-Economic Indexes for Areas (SEIFA), Australia - Data only Canberra: Australian Bureau of Statistics 2013. Available from:

http://www.abs.gov.au/AUSSTATS/abs@.nsf/DetailsPage/2033.0.55.0012011?Open Document.

20. Bekker J, Bruck D, Sciberras E. Congruent Validity of the Strength and Difficulties Questionnaire to Screen for Comorbidities in Children With ADHD. J Atten Disord. 2013.

21. Sawyer MG RJ, Arney FM, Whitham JN, Clark JJ, Baghurst PA. . Use of health and school-based services in Australia by young people with attentiondeficit/hyperactivity disorder. J Amer Acad Child Adolesc Psychiatry. = 2004;43(11):1355-63.

22. Wolraich ML, Lambert EW, Bickman L, Simmons T, Doffing MA, Worley KA. Assessing the impact of parent and teacher agreement on diagnosing attention-deficit hyperactivity disorder. J Dev Behav Pediatr. 2004;25(1):41-7.

23. Hamrin V, McCarthy EM, Tyson V. Pediatric psychotropic medication initiation and adherence: a literature review based on social exchange theory. J Child Adolesc Psychiatr Nurs. 2010 23(3):151-72.

24. Sinha D, Efron D. Complementary and alternative medicine use in children with attention deficit hyperactivity disorder. J Paediatr Child Health 2005;41(1):23-6.

25. Hazell PL, McDowell MJ, Walton JM. Management of children prescribed psychostimulant medication for attention deficit hyperactivity disorder in the Hunter region of NSW. Med J Aust. 1996;165(9):477-80.

26. Epstein JN, Langberg JM, Lichtenstein PK, Mainwaring BA, Luzader CP, Stark LJ. Community-wide intervention to improve the attention-deficit/hyperactivity disorder assessment and treatment practices of community physicians. Pediatrics.

2008;122(1):19-27.

27. Epstein JN, Langberg JM, Lichtenstein PK, Altaye M, Brinkman WB, House K, et al. Attention-Deficit/Hyperactivity Disorder Outcomes for Children Treated in Community-Based Pediatric Setting. Arch Pediatr Adolesc Med. 2010;164(2):160-5.

28. Conners CK. Conners 3rd edition. Multi-Health Systems (eds). 2008.

29. Goodman R. The Strengths and Difficulties Questionnaire: A Research Note. J Child Psychol Psychiatry. 1997;38:581-6.

30. Sung V, Hiscock H, Sciberras E, Efron D. Sleep problems in children with attentiondeficit/hyperactivity disorder: prevalence and the effect on the child and family. Arch Pediatr Adolesc Med. 2008;162(4):336-42.

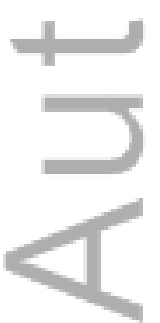

This article is protected by copyright. All rights reserved. 


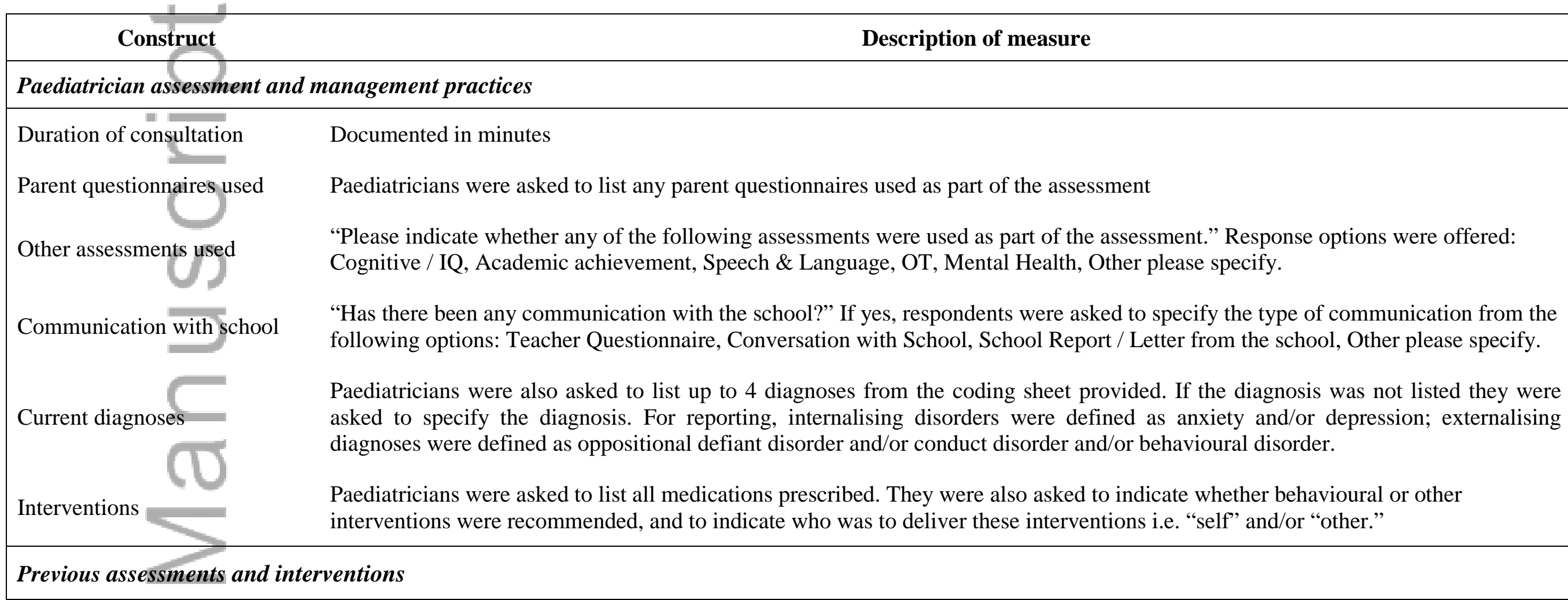

Raised the possibility of ADHD

Help sought prior to diagnosis

ADHD criteria research
Parents were asked "Who first raised the possibility of ADHD in child?" The following response options were offered: Self, Partner, Relative/Friend, teacher, Health professional, Sporting or club instructor/supervisor, Other please specify

"Have you seen any professionals previously for help with your child's behaviour and/or learning?" The following response options were offered: psychologist, general practitioner, paediatrician, child psychiatrist, speech pathologist, special educator/tutor, social worker/family support worker, alternative therapist e.g. naturopath, chiropractor, other please specify.

"Prior to the diagnosis, had you researched the ADHD diagnostic criteria?" (Yes/No) 
Paediatrician consultation timing and visits

.

Complementary/alternative therapies and food restriction

\section{Parent report of child's symptoms / problems, agreement with ADHD diagnosis}

\section{ADHD symptoms}

10-item validated Conners 3 ADHD Index (Conners 3AI), ${ }^{28}$ which has a population mean of 50 and a standard deviation of 10.

Internalising, externalising comorbidities

Sleep problems

\section{ms}

Agreement with paediatrician diagnosis
"How long did it take from when you were first concerned about your child to seeking referral for a paediatric assessment?" "After receiving a referral, how long did you have to wait to see a paediatrician?"

"How many consultations did it take for the paediatrician to diagnose your child with ADHD?"

"Does your child use any natural or complementary therapies (e.g. fish oil, chiropractor, neurofeedback) for learning, emotional or behavioural problems?"

"Do you restrict foods from your child's diet to improve their learning, behaviour or emotional difficulties (e.g. additives and colourings)?"
Assessed using the Strengths and Difficulties Questionnaire (SDQ) ${ }^{29}$ Internalising and externalising comorbidities were defined as clinical range scores on the Emotional Problems and Conduct Problems subscales (5-items each) respectively.

Sleep problem severity over the past 4 weeks was rated on a 4-point scale from "no problem" to a "large problem" and dichotomized no/mild versus moderate/severe. ${ }^{30}$

Parents were asked whether they agree with the diagnosis (5-point item from "Completely agree" to "Completely disagree"). 
Table 2. Characteristics of consultations where children were diagnosed with ADHD

Consultation characteristics $(n=137) \quad n^{*}\left(\% ; 95 \% \mathbf{C I}^{\mathrm{a}}\right)$

Duration of visit in minutes, mean (SD; 95\% CI)

$47.5(21.2 ; 38.4,56.6)$

Measurements taken, $\mathrm{n}(\%)$

Height

$126(92.0 ; 81.0,96.9)$

Weight

$130(94.9 ; 86.9,98.1)$

Blood pressure

$75(72.1 ; 39.2,90.3)^{\mathrm{b}}$

Assessments, n (\%)

Communication with school

$129(94.2 ; 82.7,98.2)$

Teacher questionnaire

$117(85.4 ; 68.6,94.0)$

Conversation with school

$21(15.3 ; 7.5,28.9)$

School report/letter from school

$90(65.7 ; 48.6,79.5)$

Other type of communication with school

$9(6.6 ; 3.1,13.6)$

Parent Questionnaire

$121(88.3 ; 74.5,95.1)$

Other assessments, n (\%)

Cognitive / IQ

$95(69.3 ; 52.6,82.2)$

Academic Achievement

$72(52.6 ; 31.2,73.0)$

Language

$56(40.9 ; 29.4,53.5)$

Occupational Therapist

$43(31.4 ; 21.4,43.5)$

Mental Health

$37(27.0 ; 13.9,45.8)$

Other

$22(16.1 ; 10.3,24.1)$

No. of comorbid diagnoses

0

$41(29.9 ; 18.7,44.2)$

1

$53(38.7 ; 30.7,47.3)$

$2+$

$43(31.4 ; 17.7,49.3)$

This article is protected by copyright. All rights reserved. 
Comorbid diagnoses

Learning disability

$36(26.3 ; 18.1,36.5)$

Anxiety

$25(18.3 ; 8.3,35.6)$

Oppositional defiant disorder

$16(11.7 ; 6.4,20.4)$

Language delay

$16(11.7 ; 5.3,23.9)$

Autism spectrum disorder

$14(10.2 ; 6.0,16.9)$

Developmental delay

$6(4.4 ; 1.8,10.1)$

Sleep disorder

$5(3.7 ; 1.3,10.2)$

Enüresis

$4(2.9 ; 1.1,7.6)$

Behaviour (eg. temper tantrums, rages)

$3(2.2 ; 0.6,8.09)$

Chromosome/Genetic disorder

$3(2.2 ; 0.7,6.5)$

Conduct disorder

$2(1.5 ; 0.4,5.8)$

Interventions, n (\%)

Medication related to ADHD

$102(74.5 ; 67.4,85.0)$

Methylphenidate

$96(70.1 ; 58.8,79.4)$

Dexamphetamine

$5(3.7 ; 1.2,10.2)$

Atomoxetine

$1(0.7 ; 0.1,6.0)$

Behavioural

$106(77.4 ; 61.4,88.0)$

$58(42.3 ; 13.8,29.2)$

$62(41.9 ; 24.2,62.8)$

$20(14.6 ; 6.5,29.5)$

Delivered by both

$7.1(6.3,8.0 ; 5.0)^{\mathrm{b}}$

Interval until next appointment, weeks, mean (95\% CI; SD)

$6.5(6.7,12.3 ; 3.9)$

Medication prescribed

$9.5(5.3,7.7 ; 7.6)$

No medication prescribed

\footnotetext{
${ }^{*}$ Unless otherwise specified

${ }^{\mathrm{a}}$ Accounting for sampling frame paediatrician clustering; ${ }^{\mathrm{b}}$ Only asked in WA $(\mathrm{n}=104)$
}

This article is protected by copyright. All rights reserved. 
Table 3. Parental report of path to diagnosis, child symptoms and agreement with diagnosis

Parent report $(\mathbf{n}=\mathbf{8 0})$ $\mathbf{n}(\%)^{*}$

\section{Path to diagnosis}

Person who first raised the possibility of ADHD

Health professional

Self

$23(29.5)$

Teacher

$20(25.6)$

Relative/Friend

Partner

Use of dietary and complementary therapies

Natural / complementary therapies

Restricted diet

Either natural / complementary therapies or restricted diet

Previously seen professionals child's behaviour and/or learning

Researched ADHD diagnostic criteria prior to diagnosis

$40(67)^{\mathrm{a}}$

Elapsed time between, (months), mean (SD)

Parent concern and seeking a referral

Interval from referral to first consultation

No. of paediatric consultations to reach ADHD diagnosis

1

2

$3+$

\section{Child symptoms}

Conners 3AI (t-score), mean (SD)

$81.4(14.9)$ 
Emotional problems

$40(50.6)$

Behavioural problems

$52(65.8)$

Moderate/severe sleep problem

$30(38.5)$

\section{Agreement with ADHD diagnosis}

How closely do parents agree with the ADHD diagnosis

Completely agree

Somewhat agree

$13(16.7)$

Neutral

Somewhat disagree

Completely disagree

$4(5.1)$

${ }^{*}$ Unless otherwise specified

${ }^{a}$ Due to slight variation in each state, these questions were only asked in WA $(n=60)$

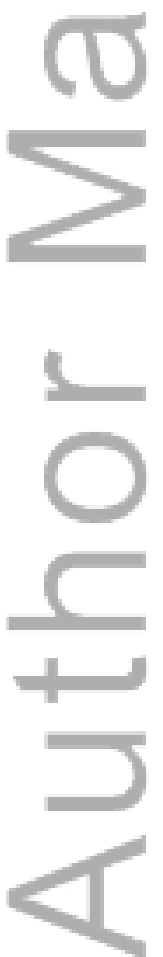

This article is protected by copyright. All rights reserved. 


\section{University Library}

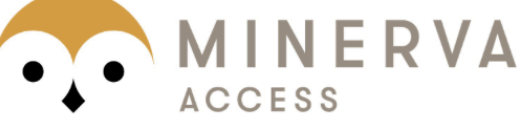

A gateway to Melbourne's research publications

Minerva Access is the Institutional Repository of The University of Melbourne

Author/s:

Efron, D;Sciberras, E;Hiscock, H;Jongeling, B;Lycett, K;Bisset, M;Smith, G

Title:

The diagnosis of attention-deficit/hyperactivity disorder in Australian children: Current paediatric practice and parent perspective

Date:

2016-04-01

Citation:

Efron, D., Sciberras, E., Hiscock, H., Jongeling, B., Lycett, K., Bisset, M. \& Smith, G. (2016). The diagnosis of attention-deficit/hyperactivity disorder in Australian children: Current paediatric practice and parent perspective. JOURNAL OF PAEDIATRICS AND CHILD HEALTH, 52 (4), pp.410-416. https://doi.org/10.1111/jpc.13091.

Persistent Link:

http://hdl.handle.net/11343/291131 